\title{
The Relationship between Pulse Wave Velocity and Coronary Artery Stenosis and Percutaneous Coronary Intervention: a retrospective observational study
}

\author{
Hyung Joon Joo, Sang-A Cho, Jae-Young Cho, Jae Hyoung Park, Soon Jun Hong, Cheol Woong Yu
} and Do-Sun Lim*

\begin{abstract}
Background: Arterial stiffness has been suggested as a valuable predictor of coronary artery stenosis (CAS). However, little data are available on aortic stiffness and CAS in patients who have previously undergone percutaneous coronary artery intervention ( $\mathrm{PCl}$ ). The aim of this study was to investigate the association of arterial stiffness to CAS in patients with a history of $\mathrm{PCl}$ and those without a history of $\mathrm{PCl}$.

Methods: We retrospectively studied 1093 consecutive patients who had undergone coronary angiography (CAG). Arterial stiffness was determined by brachial-ankle pulse wave velocity (baPWV) measured prior to CAG.

Results: In patients without a history of $\mathrm{PCl}$, baPWV significantly increased in patients with CAS compared to that in patients without CAS $(p<0.001)$. However, among patients with a history of $\mathrm{PCl}$, there was no significant difference in baPW. Multivariate logistic regression analysis demonstrated that baPWV was an independent risk predictor for CAS in patients without a history of $\mathrm{PCl}$, but not in those with a history of $\mathrm{PCI}(\mathrm{OR} 1.106,95 \% \mathrm{Cl} 1.039-1.177, p=0.002)$. In CAS patients without a history of $\mathrm{PCl}$, increased baPW was significantly associated with multiple cardiovascular risk factors, multivessel involvement, and anatomical severity.
\end{abstract}

Conclusions: Prediction of CAS by baPWW is significantly attenuated in patients with a history of PCl.

Keywords: Aortic stiffness, Pulse wave velocity, Coronary artery stenosis, Percutaneous coronary artery intervention

\section{Study highlights}

1. baPWV is significantly increased in CAS patients without a history of PCI compared to those non-CAS patients.

2. baPWV is an independent risk predictor for CAS

3. Higher baPWV is associated with multiple vessel involvement and angiographic severe disease.

4. The relationship between baPWV and CAS was attenuated in patients with abnormal ABI.

\footnotetext{
* Correspondence: dslmd@kumc.or.kr
}

Department of Cardiology, Cardiovascular Center, Korea University Anam Hospital, 126-1, 5ka, Anam-dong, Sungbuk-ku, Seoul 136-705, Republic of Korea

(c) The Author(s). 2017 Open Access This article is distributed under the terms of the Creative Commons Attribution 4.0 International License (http://creativecommons.org/licenses/by/4.0/), which permits unrestricted use, distribution, and reproduction in any medium, provided you give appropriate credit to the original author(s) and the source, provide a link to the Creative Commons license, and indicate if changes were made. The Creative Commons Public Domain Dedication waiver (http://creativecommons.org/publicdomain/zero/1.0/) applies to the data made available in this article, unless otherwise stated.

\section{Background}

Despite advances in risk stratification and its treatment, coronary artery disease remains a leading cause of morbidity and mortality worldwide. Coronary atherosclerosis and subsequent coronary plaque development are the main pathognomonic signs of coronary artery disease. Thus, early detection of coronary plaque and coronary artery stenosis (CAS) is important for the management of this disease.

Coronary angiography (CAG) continues to be considered the gold standard procedure for the diagnosis of CAS. However, its invasiveness limits its clinical use exclusively to subjects with a high-risk profile for CAS. Although many non-invasive techniques may be used to detect CAS, including the treadmill test, stress echocardiography, myocardial perfusion scanning, and computed 
tomographic angiography, these too have limitations when applied to asymptomatic subjects or subjects with a lowrisk profile.

Recently, arterial stiffness has emerged as a potential candidate for the prediction of cardiovascular disease [1-3]. It too is associated with other cardiovascular risk factors, including age, hypertension and diabetes mellitus [4-6]. Various methods have been employed to measure arterial stiffness. Among them, pulse wave velocity (PWV) and the augmentation index (AIx) have been frequently used as reliable indicators of arterial stiffness [7]. Brachial-ankle pulse wave velocity (baPWV) can be measured non-invasively to estimate the conventional carotid-femoral PWV [8]. However, the association of baPWV or AIx with CAS is still controversial. Some studies have reported that baPWV is a meaningful risk predictor for CAS $[9,10]$. In contrast, other studies have reported the limited value of baPWV to predict CAS [11]. Moreover, the discrepancy of baPWV and AIx for CAS has been also reported $[12,13]$. At present, no data are available on baPWV and AIx in patients who have previously undergone percutaneous coronary intervention (PCI).

The purpose of the present study was to explore the association between arterial stiffness (measured by PWV and AIx) and CAS in patients who have already undergone percutaneous coronary intervention (PCI) as compared to that in patients without a history of $\mathrm{PCI}$ treatment.

\section{Methods}

\section{Study design}

This was a single-center, retrospective study of consecutive patients who underwent both CAG and arterial stiffness measurements from January 2011 through January 2013. It was approved by the ethical committee of the institutional review board of Korea University Anam Hospital, and the need for written informed consent was waived due to the non-interventional and retrospective nature of the study. After excluding the patients with acute coronary syndrome, underlying valvular heart disease or prior surgical coronary revascularization, a total of 1093 patients were identified and divided into 4 groups as follows: (Group 1, $n=326$ ) absence of CAS without prior PCI history, (Group 2, n=457) presence of CAS without prior PCI history, (Group 3, $n=120$ ) absence of CAS with prior PCI history, and (Group 4, $n=$ 190) presence of CAS with prior PCI history (Fig. 1).

\section{Coronary artery stenosis (CAS)}

CAS was angiographically defined as a $>50 \%$ diameter stenosis in at least one major coronary artery (left anterior descending artery, left circumflex artery, or right coronary artery). The extent of vessel involvement was

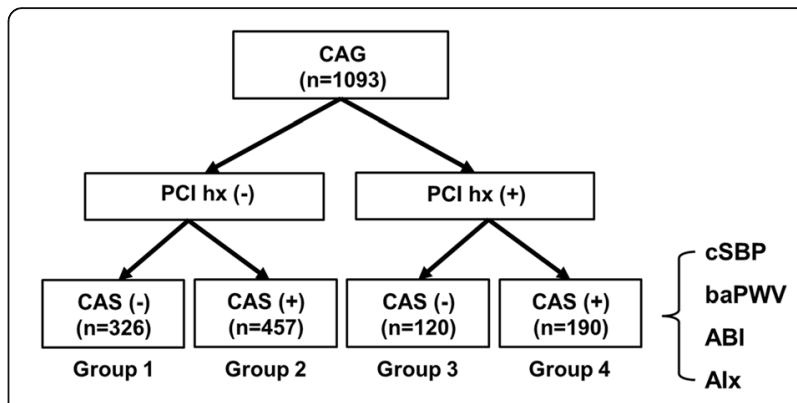

Fig. 1 Study design. CAG: coronary angiography, PCl: percutaneous coronary angiography, CAS: coronary artery stenosis, CSBP: central systolic blood pressure, baPW: brachial-ankle pulsed wave velocity, ABI: ankle-brachial index, Alx: augmentation index

divided into 0-, 1-, 2-, and 3-vessel groups. The anatomical severity of CAS was determined by using the modified Gensini score [14].

\section{Non-invasive hemodynamic parameter measurement}

baPWV and ankle-brachial index (ABI) were measured non-invasively using an oscillometric sphygmomanometric device (VP-1000 plus; Omron Colin, Kyoto, Japan). Central systolic blood pressure (cSBP) and AIx were also measured non-invasively by using an applanation tonometry device (HEM9000A1; Omron Colin, Kyoto, Japan). cSBP was estimated from the late systolic shoulder (SBP2) of the radial pulse waveform. Because AIx is significantly influenced by heart rate, an index normalized for a heart rate of 75 bpm (AIx@75) was used. All these measurements were performed the day prior to CAG. The validity and reproducibility of the measurements have been previously described [8, 15].

\section{Statistical analysis}

Continuous variables were expressed as the mean \pm SD. Comparisons between the 2 groups were performed using an independent Student's $t$-test for continuous variables and $X^{2}$ test for categorical variables. To identify the risk predictors for CAS, selected factors from all variables were tested using a multivariate logistic regression analysis by univariate analysis $(p<0.10)$. An odds ratio with a $95 \%$ confidence interval and $P$-value were reported. Receiver operating characteristic (ROC) curves were constructed for the diagnosis of significant coronary artery stenosis. Two academic authors separately analyzed the database and reconciled any discrepancies. All tests were two-sided. A $P$-value of less than 0.05 was considered statistically significant. All statistical analyses were performed using the Statistical Package for the Social Sciences (SPSS) software package (Version 18.0, SPSS Inc., Chicago, IL, USA). 


\section{Results}

\section{Baseline demographic characteristics}

Demographic features of study subjects are presented in Table 1. Patients with a history of PCI (Groups 3 and 4) were significantly older, predominantly male, and presented with a higher incidence of typical or atypical chest pain, hypertension, diabetes, dyslipidemia, and impaired renal function compared to the patients without a history of PCI (Groups 1 and 2). Patients with a history of PCI were more frequently receiving ACE inhibitors, angiotensin receptor blockers, beta-blockers, calcium channel blockers, insulin and statins than patients with no history of PCI. However, among the patients without a history of PCI, the patients with CAS (Group 2) had more typical chest pain compared to those without CAS (Group 1). Among patients with a history of PCI, patients without CAS (Group 3) were older and had a higher incidence of diabetes than patients with CAS (Group 4).

\section{Non-invasive hemodynamic parameters and CAS}

Non-invasive hemodynamic parameters are summarized in Table 2. Among patients without a history of PCI, those with CAS (Group 2) had a significantly lower diastolic blood pressure (DBP), higher pulse pressure, lower cSBP, higher baPWV, and lower ABI than patients

Table 1 Baseline demographic characteristics

\begin{tabular}{|c|c|c|c|c|c|c|c|}
\hline Variables & Group 1 & Group 2 & $\begin{array}{l}p \text {-value } \\
(1 \text { vs } 2)\end{array}$ & Group 3 & Group 4 & $\begin{array}{l}p \text {-value } \\
(3 \text { vs } 4)\end{array}$ & $\begin{array}{l}p \text {-value } \\
(1 / 2 \text { vs } 3 / 4)\end{array}$ \\
\hline Age (year) & $58.9 \pm 10.04$ & $59 \pm 10.89$ & 0.896 & $63.88 \pm 8.32$ & $61.41 \pm 10.04$ & 0.020 & $<0.001$ \\
\hline Male (\%) & 182 (55.83\%) & 259 (56.67\%) & 0.814 & $84(70 \%)$ & 148 (77.89\%) & 0.119 & $<0.001$ \\
\hline \multicolumn{8}{|l|}{ Symptoms } \\
\hline Asymptomatic & $70(20.5 \%)$ & $66(14.4 \%)$ & 0.001 & $75(62.5 \%)$ & $96(50.5 \%)$ & 0.078 & $<0.001$ \\
\hline Atypical chest pain & 127 (39.0\%) & 149 (32.6\%) & & $15(12.5 \%)$ & $24(12.6 \%)$ & & \\
\hline Typical chest pain & 129 (39.6\%) & $242(53.0 \%)$ & & $30(25.0 \%)$ & 70 (36.8\%) & & \\
\hline $\mathrm{BMI}(\mathrm{kg} / \mathrm{m} 2)$ & $24.9 \pm 3.09$ & $24.71 \pm 2.97$ & 0.391 & $24.68 \pm 2.8$ & $24.99 \pm 3.04$ & 0.356 & 0.683 \\
\hline Smoking (\%) & $97(29.75 \%)$ & $138(30.2 \%)$ & 0.894 & $38(31.67 \%)$ & $51(26.84 \%)$ & 0.360 & 0.671 \\
\hline Hypertension (\%) & 191 (58.4\%) & $266(58.3 \%)$ & 1.000 & 108 (90.0\%) & $180(94.7 \%)$ & 0.172 & $<0.001$ \\
\hline Diabetes mellitus (\%) & $61(18.71 \%)$ & $82(17.94 \%)$ & 0.784 & $46(38.33 \%)$ & $50(26.32 \%)$ & 0.026 & $<0.001$ \\
\hline Dyslipidemia (\%) & $95(29.14 \%)$ & 149 (32.6\%) & 0.302 & $102(85 \%)$ & 164 (86.32\%) & 0.746 & $<0.001$ \\
\hline CKD (\%) & $69(21.23 \%)$ & $110(24.07 \%)$ & 0.352 & $42(35 \%)$ & $53(27.89 \%)$ & 0.186 & 0.008 \\
\hline Uric acid (mg/dL) & $5.14 \pm 1.48$ & $5.25 \pm 1.44$ & 0.337 & $5.3 \pm 1.63$ & $5.42 \pm 1.34$ & 0.493 & 0.085 \\
\hline Hba1c (\% of THb) & $6.19 \pm 1.04$ & $6.29 \pm 1.41$ & 0.539 & $6.48 \pm 1.08$ & $6.02 \pm 0.82$ & 0.005 & 0.644 \\
\hline $\mathrm{TC}(\mathrm{mg} / \mathrm{dL})$ & $147.8 \pm 89.48$ & $157.3 \pm 103.2$ & 0.168 & $123.7 \pm 63.19$ & $133.7 \pm 80.2$ & 0.226 & $<0.001$ \\
\hline LDL-C (mg/dL) & $96.5 \pm 26.88$ & $96.13 \pm 27.24$ & 0.853 & $72.64 \pm 20.31$ & $73.07 \pm 21.8$ & 0.860 & $<0.001$ \\
\hline $\mathrm{HDL}-\mathrm{C}(\mathrm{mg} / \mathrm{dL})$ & $51.31 \pm 13.57$ & $49.95 \pm 12.83$ & 0.157 & $48.1 \pm 11.29$ & $46.89 \pm 11.36$ & 0.361 & $<0.001$ \\
\hline Triglyceride (mg/dL) & $137.5 \pm 89.21$ & $152.3 \pm 105.9$ & 0.036 & $119.6 \pm 67.36$ & $129.9 \pm 82.59$ & 0.231 & $<0.001$ \\
\hline Creatinine (mg/dL) & $0.96 \pm 0.46$ & $0.95 \pm 0.23$ & 0.672 & $1.06 \pm 0.65$ & $1.02 \pm 0.2$ & 0.479 & 0.003 \\
\hline hsCRP (mg/L) & $2.74 \pm 8.99$ & $2.15 \pm 5.68$ & 0.324 & $2.01 \pm 4.32$ & $1.82 \pm 4$ & 0.701 & 0.162 \\
\hline$\#$, risk factors ${ }^{a}$ & $2.23 \pm 1.39$ & $2.29 \pm 1.35$ & 0.579 & $3.67 \pm 1.25$ & $3.48 \pm 0.97$ & 0.152 & $<0.001$ \\
\hline \multicolumn{8}{|l|}{ Index Medications } \\
\hline ACE inhibitors (\%) & $12(3.68 \%)$ & $15(3.28 \%)$ & 0.763 & $35(29.17 \%)$ & $55(28.95 \%)$ & 0.967 & $<0.001$ \\
\hline ARBs (\%) & $87(26.69 \%)$ & $101(22.1 \%)$ & 0.139 & $52(43.33 \%)$ & $68(35.79 \%)$ & 0.184 & $<0.001$ \\
\hline Beta-blockers (\%) & $35(10.74 \%)$ & $62(13.57 \%)$ & 0.236 & $47(39.17 \%)$ & $88(46.32 \%)$ & 0.216 & $<0.001$ \\
\hline CCB (\%) & $84(25.77 \%)$ & 135 (29.54\%) & 0.246 & 47 (39.17\%) & $83(43.68 \%)$ & 0.432 & $<0.001$ \\
\hline Diuretics (\%) & $27(8.28 \%)$ & $28(6.13 \%)$ & 0.245 & $12(10 \%)$ & 15 (7.89\%) & 0.522 & 0.340 \\
\hline Insulin (\%) & $2(0.61 \%)$ & $2(0.44 \%)$ & 1.000 & $4(3.33 \%)$ & $5(2.63 \%)$ & 0.739 & 0.003 \\
\hline Statins (\%) & 77 (23.62\%) & $116(25.38 \%)$ & 0.573 & $102(85 \%)$ & 163 (85.79\%) & 0.848 & $<0.001$ \\
\hline
\end{tabular}

$B M I$ body mass index, $C K D$ chronic kidney disease, $T C$ total cholesterol, $L D L-C$ low-density lipoprotein cholesterol, $H D L-C$ high-density lipoprotein cholesterol, $A C E$ angiotensin-converting enzyme, $A R B$ angiotensin receptor blocker, $C C B$ calcium channel blocker

${ }^{a}$ Risk factors included old age ( $\geq 65$ years), male gender, smoking, hypertension, diabetes mellitus, and dyslipidemia. Data are presented as means \pm SDs for continuous variables and numbers (\%) for categorical variables 
Table 2 Comparisons of SBP, DBP, pulse pressure, CSBP, baPWV, ABI, Aix, and Alx@75

\begin{tabular}{lccccccc}
\hline Variables & Group 1 & Group 2 & $\begin{array}{l}p \text {-value } \\
(1 \text { vs 2) }\end{array}$ & Group 3 & Group 4 & $\begin{array}{c}p \text {-value } \\
(3 \text { vs 4) }\end{array}$ & $\begin{array}{c}p \text {-value } \\
(1 / 2 \text { vs 3/4) }\end{array}$ \\
\hline SBP (mmHg) & $130.3 \pm 17.72$ & $130.7 \pm 17.12$ & 0.762 & $129.2 \pm 15.15$ & $131.9 \pm 17.25$ & 0.196 & 0.804 \\
DBP (mmHg) & $77.56 \pm 10.77$ & $74.12 \pm 11.76$ & $<0.001$ & $78.34 \pm 9.98$ & $76.26 \pm 11.8$ & 0.141 & 0.067 \\
Pulse pressure (mmHg) & $52.75 \pm 13.95$ & $56.6 \pm 13.37$ & $<0.001$ & $50.84 \pm 12.74$ & $55.63 \pm 13.45$ & 0.004 & 0.222 \\
CSBP (mmHg) & $135.2 \pm 20.99$ & $134.2 \pm 19.33$ & 0.529 & $134.5 \pm 18.23$ & $136.3 \pm 19.71$ & 0.467 & 0.488 \\
baPWV (right, cm/sec) & $1518.6 \pm 292.1$ & $1607.4 \pm 342.6$ & $<0.001$ & $1521.4 \pm 241.3$ & $1572.4 \pm 339.3$ & 0.124 & 0.409 \\
baPWV (left, cm/sec) & $1517.4 \pm 292.0$ & $1592.4 \pm 335.5$ & 0.001 & $1533.0 \pm 250.9$ & $1559.6 \pm 319.0$ & 0.416 & 0.574 \\
baPWV (average, cm/sec) & $1517.7 \pm 289.1$ & $1599.8 \pm 326.7$ & $<0.001$ & $1526.8 \pm 241.6$ & $1566.0 \pm 323.8$ & 0.226 & 0.476 \\
ABI (right) & $1.13 \pm 0.09$ & $1.12 \pm 0.1$ & 0.142 & $1.12 \pm 0.08$ & $1.12 \pm 0.11$ & 0.795 & 0.343 \\
ABI (left) & $1.13 \pm 0.08$ & $1.1 \pm 0.12$ & $<0.001$ & $1.12 \pm 0.08$ & $1.11 \pm 0.11$ & 0.571 & 0.604 \\
ABI (average) & $1.13 \pm 0.07$ & $1.11 \pm 0.1$ & 0.007 & $1.12 \pm 0.08$ & $1.12 \pm 0.11$ & 0.700 & 0.820 \\
Alx (\%) & $81.73 \pm 14.64$ & $80.96 \pm 14.36$ & 0.492 & $82.19 \pm 16.25$ & $81.37 \pm 13.8$ & 0.661 & 0.700 \\
Alx@75 (\%) & $79.8 \pm 13.26$ & $78.11 \pm 12.5$ & 0.090 & $80.58 \pm 14.02$ & $79.23 \pm 11.81$ & 0.402 & 0.314 \\
\hline Data are prst & & & & &
\end{tabular}

Data are presented as means \pm SDs

$S B P$ systolic blood pressure, DBP diastolic blood pressure, $C S B P$ central systolic blood pressure, baPWV brachial-ankle pulsed wave velocity, $A B I$ ankle-brachial index, Alx augmentation index, Alx@75 Alx normalized for a heart rate of 75 bpm

without CAS (Group 1). However, among patients with a history of PCI, those with CAS (Group 4) had a significantly higher pulse pressure than those without CAS (Group 3).

To explore the potential risk predictors for CAS, we performed univariate and multivariate logistic regression analyses (Table 3). In the total population, the use of RAS inhibitors and statins, DBP, cSBP, baPWV, ABI, and AIx@75 were independent risk predictors for CAS. In patients without a history of $\mathrm{PCI}$, hypertension, the use of CCB, DBP, cSBP, baPWV, ABI, and AIx@75 were independent risk predictors for CAS. In patients with a history of PCI, diabetes mellitus, DBP, cSBP, and AIx@75 were independent risk predictors for CAS.

Thus, baPWV significantly increased in CAS patients without a history of PCI as an independent risk predictor for CAS.

\section{baPWV and coronary artery disease risk factors}

Next, we compared baPWVs dependent to the presence or absence of conventional cardiovascular risk factors, which included age ( $\geq 65$ years), gender, smoking, hypertension, type 2 diabetes mellitus, and dyslipidemia (Fig. 2a). Among patients without a history of PCI, baPWV increased significantly in smokers than in non-smokers $(p=0.03)$. baPWV was also higher in male patients than female patients, although this difference was not significant $(p=0.10)$. Among patients with a history of PCI, baPWV decreased significantly in diabetic patients than in non-diabetic patients $(p=$ 0.03). Interestingly, among patients without a history of PCI, baPWV increased significantly in patients with CAS or multiple conventional coronary artery disease risk factors (Fig. 2b). However, among patients without a history of PCI, the number of conventional coronary artery risk factors or CAS did not affect baPWV. These data suggest that increased baPWV in CAS patients without a history of PCI could be associated with an overall increase in cardiovascular risk.

\section{baPWV and the severity of CAS}

Although baPWV did not significantly differ with the numbers of stenotic major coronary arteries in patients with a history of PCI, baPWV increased significantly in cases of 2-vessel or greater involvement in patients without a history of PCI (Fig. 3, left panel). Patients were divided into 4 groups according to their modified Gensini score: Q1 = zero, Q2 $=0.5$ to 9.5, Q3 $=10$ to 29.5 and $\mathrm{Q} 4=30$ and over. In both patients with and without a history of PCI, baPWVs in Q2, Q3, and Q4 were significantly higher than those in Q1 (Fig. 3, right panel). This suggests that baPWV increased significantly even in patients with mild coronary artery stenosis (the modified Gensini score $>0.5$ ), regardless of a history of PCI. Interestingly, in patients without a history of PCI, baPWV in Q4 was significantly higher than that in Q3 $(p=0.039)$. When taken in conjunction with the extent of vessel involvement, these data suggest that baPWV showed a trend towards a positive correlation with anatomical severity and multivessel involvement in patients without a history of PCI.

\section{Effect of $A B I$ on baPWV and CAS}

$\mathrm{ABI}$ is a well-known indicator for peripheral artery disease [16]. The reference value for $\mathrm{ABI}$ is known to be 1.0-1.29. A value of less than 0.9 suggests significant stenosis of the lower limb artery. A value greater than 
Table 3 Univariate and multivariate analyses for coronary artery stenosis (CAS)

\begin{tabular}{|c|c|c|c|c|c|c|}
\hline \multirow[t]{2}{*}{ Variable } & \multicolumn{3}{|c|}{ Univariate analysis } & \multicolumn{3}{|c|}{ Multivariate analysis } \\
\hline & $\operatorname{Exp}(B)$ & OR $(95 \% \mathrm{Cl})$ & $p$-value & $\operatorname{Exp}(B)$ & OR $(95 \% \mathrm{Cl})$ & $p$-value \\
\hline \multicolumn{7}{|l|}{ Total population } \\
\hline Age & 0.995 & $0.983-1.007$ & 0.403 & & & \\
\hline Male & 1.148 & $0.896-1.470$ & 0.276 & & & \\
\hline BMI & 0.995 & $0.956-1.036$ & 0.808 & & & \\
\hline Smoking & 0.951 & $0.730-1.237$ & 0.706 & & & \\
\hline Hypertension & 0.840 & $0.659-1.071$ & 0.159 & & & \\
\hline Diabetes mellitus & 0.812 & $0.608-1.085$ & 0.159 & & & \\
\hline Dyslipidemia & 1.184 & $0.930-1.509$ & 0.171 & & & \\
\hline CKD & 1.013 & $0.767-1.339$ & 0.926 & & & \\
\hline RAS inhibitor & 0.843 & $0.658-1.080$ & 0.177 & 0.680 & $0.497-0.931$ & 0.016 \\
\hline Beta-blocker & 1.340 & $0.991-1.811$ & 0.057 & & & \\
\hline $\mathrm{CCB}$ & 1.222 & $0.941-1.586$ & 0.132 & & & \\
\hline Statin & 1.131 & $0.885-1.445$ & 0.325 & 1.477 & $1.084-2.014$ & 0.014 \\
\hline SBP & 1.004 & $0.996-1.011$ & 0.356 & & & \\
\hline DBP & 0.977 & $0.965-0.988$ & $<0.001$ & 0.951 & $0.935-0.967$ & $<0.001$ \\
\hline PP & 1.023 & $1.013-1.034$ & $<0.001$ & & & \\
\hline CSBP & 0.999 & $0.993-1.006$ & 0.876 & 1.021 & $1.008-1.033$ & 0.001 \\
\hline baPW & 1.001 & $1.000-1.001$ & $<0.001$ & 1.075 & $1.021-1.133$ & 0.006 \\
\hline$A B \mid$ & 0.202 & $0.053-0.774$ & 0.020 & 0.094 & $0.019-0.468$ & 0.004 \\
\hline Alx@75 & 0.990 & $0.980-1.001$ & 0.064 & 0.976 & $0.963-0.989$ & $<0.001$ \\
\hline \multicolumn{7}{|c|}{ Patients without prior $\mathrm{PCl}$ history } \\
\hline Age & 1.001 & $0.987-1.014$ & 0.895 & & & \\
\hline Male & 1.035 & $0.777-1.378$ & 0.814 & & & \\
\hline BMI & 0.980 & $0.935-1.027$ & 0.390 & & & \\
\hline Smoking & 1.021 & $0.749-1.393$ & 0.894 & & & \\
\hline Hypertension & 0.796 & $0.599-1.059$ & 0.118 & 0.667 & $0.462-0.964$ & 0.031 \\
\hline Diabetes mellitus & 0.950 & $0.658-1.371$ & 0.783 & & & \\
\hline Dyslipidemia & 1.176 & $0.864-1.602$ & 0.303 & & & \\
\hline CKD & 1.176 & $0.836-1.655$ & 0.352 & & & \\
\hline RAS inhibitor & 0.806 & $0.586-1.108$ & 0.183 & & & \\
\hline Beta-blocker & 1.305 & $0.839-2.029$ & 0.237 & & & \\
\hline $\mathrm{CCB}$ & 1.208 & $0.878-1.662$ & 0.247 & 1.552 & $1.032-2.333$ & 0.035 \\
\hline Statin & 1.100 & $0.790-1.532$ & 0.573 & & & \\
\hline SBP & 1.001 & $0.993-1.010$ & 0.762 & & & \\
\hline DBP & 0.974 & $0.961-0.987$ & $<0.001$ & 0.952 & $0.933-0.971$ & $<0.001$ \\
\hline PP & 1.022 & $1.010-1.034$ & $<0.001$ & & & \\
\hline CSBP & 0.998 & $0.990-1.005$ & 0.528 & 1.016 & $1.001-1.030$ & 0.034 \\
\hline baPWV & 1.001 & $1.000-1.001$ & $<0.001$ & 1.106 & $1.039-1.177$ & 0.002 \\
\hline$A B \mid$ & 0.123 & $0.024-0.621$ & 0.011 & 0.039 & $0.006-0.273$ & 0.001 \\
\hline Alx@75 & 0.990 & $0.978-1.002$ & 0.091 & 0.979 & $0.963-0.995$ & 0.010 \\
\hline
\end{tabular}


Table 3 Univariate and multivariate analyses for coronary artery stenosis (CAS) (Continued)

\begin{tabular}{|c|c|c|c|c|c|c|}
\hline \multicolumn{7}{|c|}{ Patients with prior $\mathrm{PCI}$ history } \\
\hline Age & 0.972 & $0.948-0.997$ & 0.026 & & & \\
\hline Male & 1.510 & $0.898-2.539$ & 0.120 & & & \\
\hline BMI & 1.038 & $0.959-1.123$ & 0.355 & & & \\
\hline Smoking & 0.792 & $0.480-1.307$ & 0.361 & & & \\
\hline Hypertension & 0.928 & $0.578-1.488$ & 0.756 & & & \\
\hline Diabetes mellitus & 0.575 & $0.352-0.937$ & 0.027 & 0.556 & $0.318-0.970$ & 0.039 \\
\hline Dyslipidemia & 1.113 & $0.581-2.132$ & 0.747 & & & \\
\hline CKD & 0.718 & $0.440-1.174$ & 0.187 & & & \\
\hline RAS inhibitor & 0.735 & $0.450-1.198$ & 0.217 & & & \\
\hline Beta-blocker & 1.340 & $0.842-2.132$ & 0.217 & & & \\
\hline $\mathrm{CCB}$ & 1.205 & $0.757-1.919$ & 0.433 & & & \\
\hline Statin & 1.066 & $0.559-2.033$ & 0.847 & & & \\
\hline SBP & 1.010 & $0.995-1.026$ & 0.196 & & & \\
\hline DBP & 0.983 & $0.961-1.006$ & 0.142 & 0.953 & $0.923-0.984$ & 0.003 \\
\hline PP & 1.029 & $1.008-1.049$ & 0.005 & & & \\
\hline CSBP & 1.005 & $0.992-1.018$ & 0.465 & 1.032 & $1.010-1.054$ & 0.004 \\
\hline baPWV & 1.000 & $1.000-1.001$ & 0.256 & & & \\
\hline$A B \mid$ & 0.640 & $0.058-7.095$ & 0.716 & & & \\
\hline Alx@75 & 0.992 & $0.972-1.011$ & 0.401 & 0.971 & $0.947-0.996$ & 0.024 \\
\hline
\end{tabular}

RAS inhibitor refers to ACE inhibitors or ARBs

CKD chronic kidney disease, OR odds ratio, 95\% Cl 95\% confidence interval

1.3 suggests non-compressible calcified vessels. An abnormal value of ABI could indicate atherosclerosis or an arteriosclerotic change to the blood vessels [17]. In the present study, we further analyzed baPWV and CAS dependence on $\mathrm{ABI}$. Interestingly, in patients without a history of PCI, baPWV significantly increased only in CAS patients with normal ABI (Fig. 4). Abnormal ABI attenuated the baPWV increase in CAS patients. This suggests that baPWV could not predict CAS in patients with vascular atherosclerosis or arteriosclerotic changes as determined by ABI.

\section{Diagnostic potential of baPWV for CAS}

Based on the ROC curves, we analyzed the accuracy of baPWV in predicting CAS. ROC curves in patients with and without a history of $\mathrm{PCI}$ are presented in Fig. 5. The area under the curve (AUC) of baPWV in patients with a history of PCI was determined to be non-significant (AUC $=0.520, p=0.548$, while that of baPWV in patients without a history of PCI was significantly higher than the reference value $(\mathrm{AUC}=$ $0.576, p<0.001)$. Using a cut-off value of $1488.5 \mathrm{~cm} / \mathrm{s}$ for baPWV, a sensitivity of $59.6 \%$ and specificity of $50.2 \%$ were obtained. A cut-off value of $1561 \mathrm{~cm} / \mathrm{s}$ for baPWV produced a $50.8 \%$ sensitivity and a $62.5 \%$ specificity value.

\section{Discussion}

Coronary atherosclerosis and arteriosclerosis are the pathognomonic processes leading to coronary vascular injury. Arterial stiffness reflects the functional and structural vascular changes that occur during atherosclerosis and arteriosclerosis [18]. Previous studies have demonstrated that arterial stiffness is significantly associated with an increased risk of coronary artery disease $[2,19,20]$ and cardiovascular events [21, 22]. Moreover, measurement of arterial stiffness is often recommended to evaluate vascular damage in daily clinical practice. Recent guidelines have recommended carotid-femoral PWV measurement for the evaluation of asymptomatic organ damage in hypertensive patients [23]. Although carotid-femoral PWV has been used as a conventional method for the measurement of arterial stiffness, baPWV has been extensively studied as an alternative method given its ease and convenience.

Numerous studies have shown that an increased baPWV is significantly associated with the presence of CAS [9-11]. In the present study, baPWV significantly increased only in CAS patients without a history of PCI (Table 2). In addition, multivariate analyses showed baPWV to be an independent predictor for CAS only in patients without a history of PCI (Table 3). Considering that previous studies in the literature excluded patients with a history of PCI, the results of the present study correspond to those previously published. 


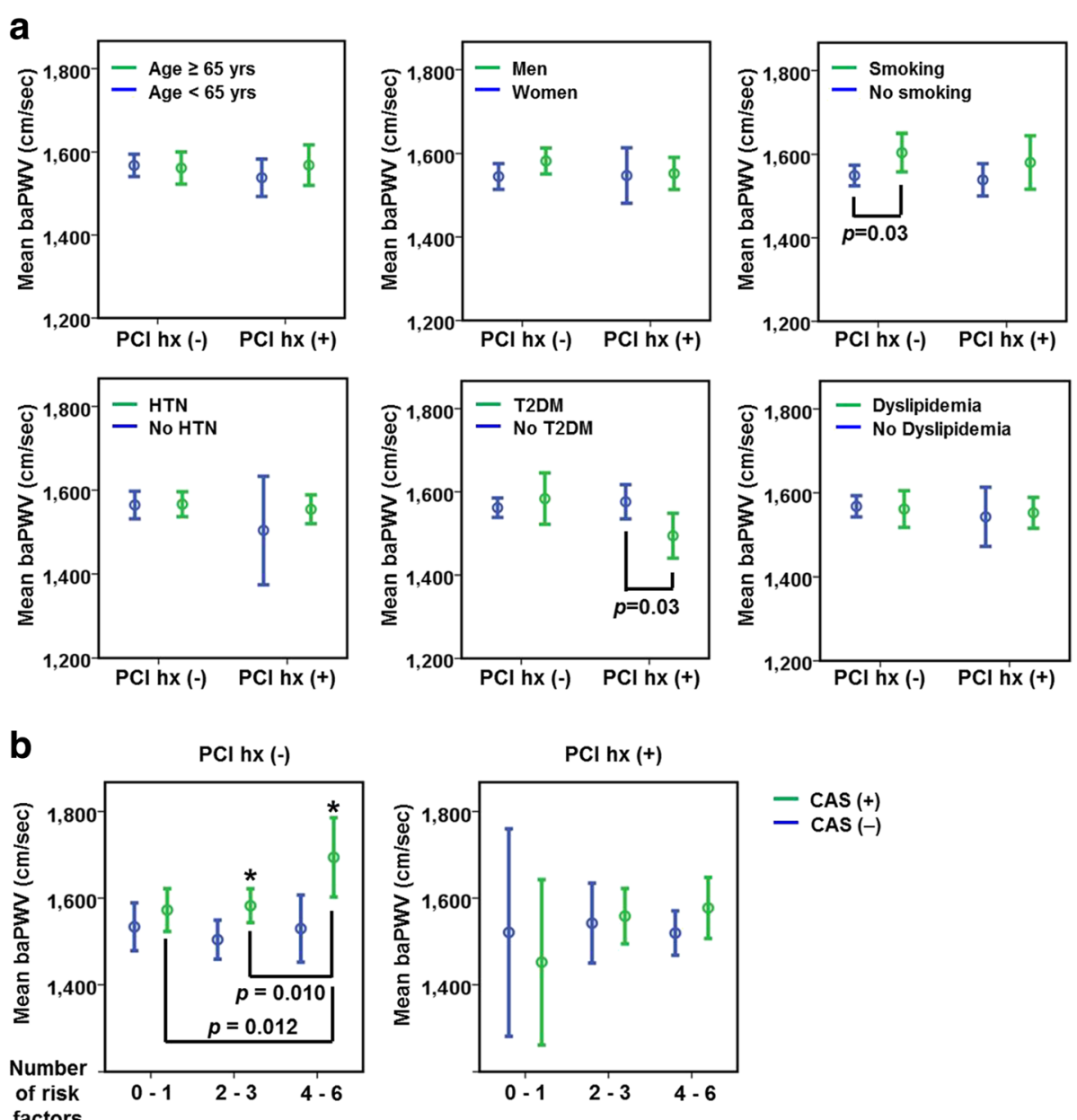

Fig. 2 baPWV and coronary artery disease risk factors. a Comparisons of baPWV according to coronary artery disease risk factors and history of PCI. $\mathbf{b}$ Comparisons of baPWW according to CAS and the number of coronary artery disease risk factors. ${ }^{*} p<0.05$ versus CAS (-). HTN: hypertension, T2DM: type 2 diabetes mellitus

Formerly, arterial stiffness was known to be associated with conventional cardiovascular risk factors, including age, hypertension, and diabetes mellitus [4-6]. The findings of the present study show that, although there were significant differences in clinical profiles (including cardiovascular risk factors) between patients with and without a history of PCI, the presence of each conventional cardiovascular risk factor (age, gender, smoking, hypertension, diabetes mellitus, and dyslipidemia) had little effect on baPWV in both patients with and without a history of PCI (Fig. 2a). Interestingly, baPWV increased in CAS patients without a history of PCI while overall cardiovascular risk increased (Fig. 2b). This association between baPWV and overall cardiovascular risk was not shown in patients without CAS or patients with a history of PCI. These findings suggests that baPWV could play a role in predicting CAS in patients at modest cardiovascular risk, such as those with more than 2 cardiovascular risk factors and no history of PCI.
In previous studies, the relationship between baPWV and the severity of CAS have shown conflicting results. Xiong et al. reported a strong relationship between baPWV and CAS severity as assessed by the SYNTAX score [24]. On the other hand, Chae et al. reported a non-significant relationship between baPWV and multivessel involvement of CAS [9]. Both studies included only patients without a history of PCI. Interestingly, the present study demonstrated that an increased baPWV was related to both multivessel involvement and the increased, modified Gensini score in patients without a history of PCI. Furthermore, this relationship was attenuated in patients with a history of PCI.

In this study, baPWV was not increased in CAS patients with a history of PCI. There are several factors to consider when explaining the relationship between baPWV and CAS and a history of PCI as observed in the present study. 

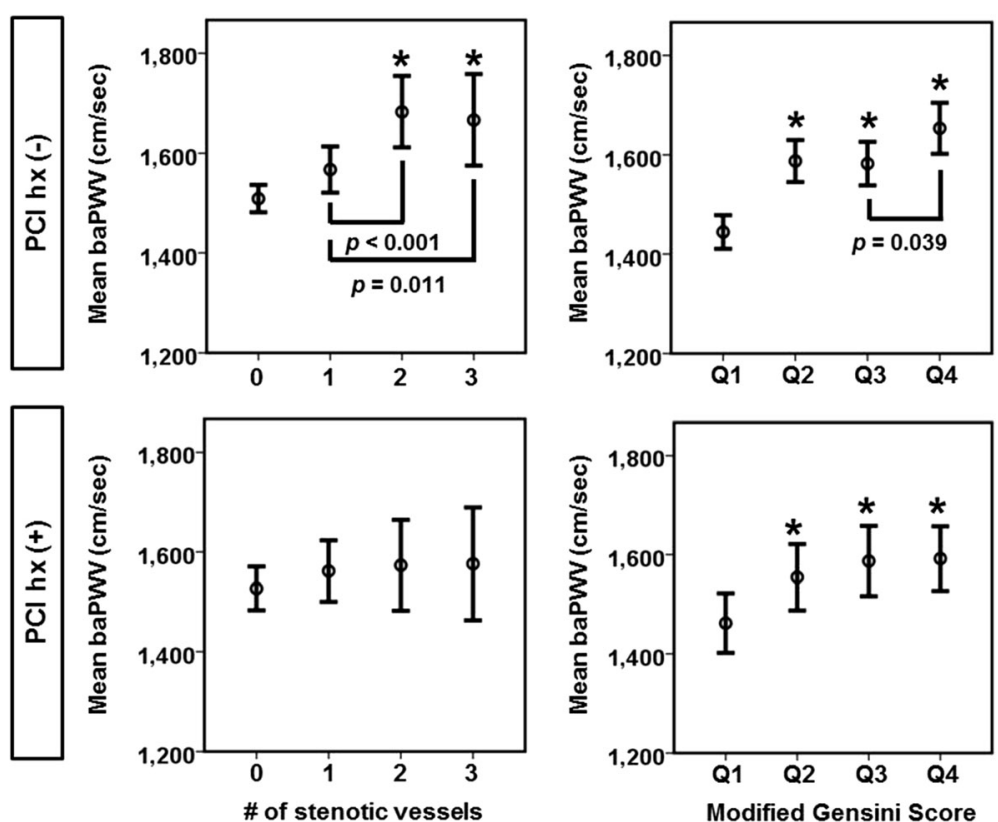

Fig. 3 baPWV and angiographical disease severity. Comparisons of baPWV according to the number of stenotic coronary arteries or the modified Gensini score. ${ }^{*} p<0.05$ versus patients with no stenotic coronary artery or the lowest modified Gensini score (Q1)

First, the baseline demographic characteristics showed significant differences between the patients with and without a history of PCI (Table 1). Patients with a history of PCI were older, male-predominant and frequently asymptomatic. These patients were also presented with an increased incidence of hypertension, diabetes, dyslipidemia and renal function impairment. The medication which was administered to these patients varied widely. Previously published studies have reported different results regarding this relationship between baPWV and CAS in different study populations. Seo et al. reported that baPWV had the limited value as a predictor of CAS in the patients with a high-risk profile [25]. Park et al.

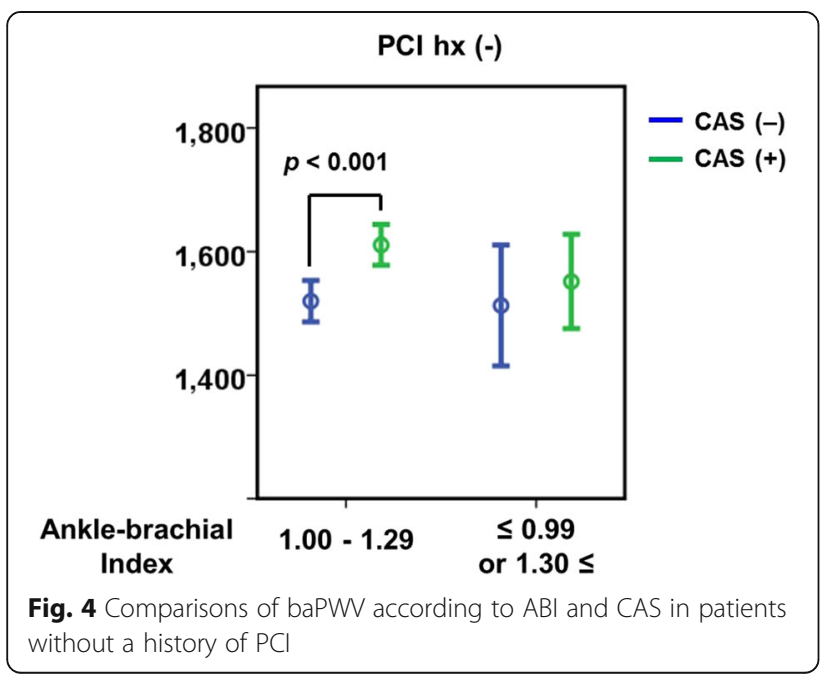

also showed that baPWV failed to predict CAS in the patients with suspected stable angina by means of a standard medical history and stress test [26]. When we analyzed the diagnostic potential of baPWV according to the pre-test probability based on the patient symptom, baPWV was significant for CAS only in asymptomatic or atypical chest pain patients without prior PCI history (Additional file 1: Table S1). It supported the previous underlying assumption that baPWV could have potential to predict CAS in low probability patients.

Second, atherosclerosis and arteriosclerosis in other types of arteries could affect baPWV and act as confounding factors in the prediction of CAS. While both conditions are known to elicit overall vascular changes as opposed to localized effects, they often cause different types of vascular diseases such as coronary artery disease and other peripheral occlusive arterial disease. ABI is thought to be reflective of atherosclerotic changes to the peripheral arteries in lower limbs $[27,28]$ and is widelyused to evaluate and diagnose peripheral artery disease [29-31]. Conventionally, a low ABI $(<0.9)$ represents significant arterial stenosis of the lower extremities and a high ABI (>1.2) represents abnormal arterial stiffness and hardening. Interestingly, although there was no significant difference in baPWV according to $\mathrm{ABI}$ and CAS in patients with a history of $\mathrm{PCI}$ in this study (data not shown), comparison of baPWV according to ABI in patients without a history of PCI did show that baPWV was elevated only in CAS patients with normal ABI (Fig. 4). This further suggests that vascular changes in 

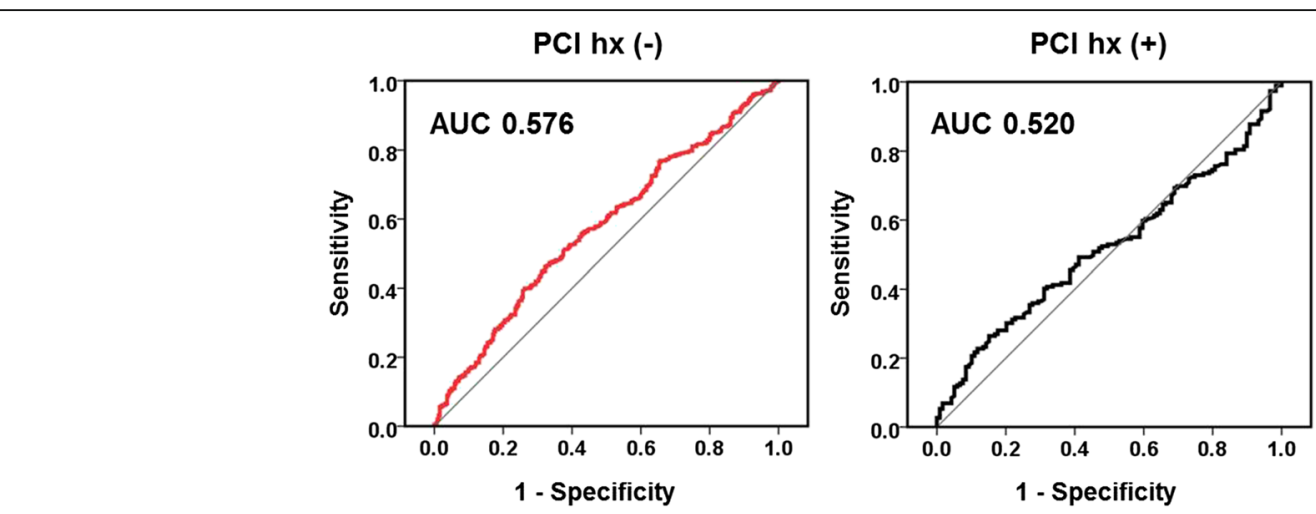

Fig. 5 ROC curves of baPWV in patients with or without a history of PCI

other arteries may have significantly limited the efficacy of baPWV in the prediction of CAS, even in patients without a history of PCI.

Finally, the present study showed that the overall diagnostic potential of baPWV for CAS, even in patients without a history of PCI, was somewhat limited. Although the AUC in the ROC curve was statistically significant, a cutoff value of $1561 \mathrm{~cm} / \mathrm{s}$ produced only a $50.8 \%$ sensitivity and a $62.5 \%$ specificity (Fig. 5). Based on previous reports, the suggested cut-off values of baPWV for the prediction of CAS vary greatly from $1540 \mathrm{~cm} / \mathrm{s}$ to $2150 \mathrm{~cm} / \mathrm{s}[9,10]$. Sensitivities and specificities for CAS in the literature are $65.0-76.7 \%$ and $56.7-61.0 \%$, respectively, which are too low to have any clinical implications. In addition, previous studies have also shown that baPWV failed to predict the risk of revascularization [9].

The limitations of this study must be taken into consideration. First, this study was a single-center, retrospective, cross-sectional study, which cannot determine causality. Second, we did not assess the treatment decisions made, including revascularization for patients with CAS. Third, although the present study successfully showed the significance of baPWV for CAS, its diagnostic potential was quite poor. To investigate its clinical implication, it should be kept being researched whether baPWV could provide the additional statistical power to the conventional patient stratification, and finally improve the overall predictability for CAS.

\section{Conclusions}

In conclusion, baPWV could play a role in predicting CAS and its severity only in patients without a history of PCI. However, its diagnostic potential for CAS is quite limited, and vascular changes in other arteries detected by ABI significantly attenuate their correlation. The clinical value of follow-up baPWV measurement in patients with CAS remains to be further investigated.

\section{Additional file}

Additional file 1: Table S1. ROC curve analysis for baPW to predict CAS. (DOCX $13 \mathrm{~kb})$

\section{Abbreviations}

ABI: Ankle-brachial index; Alx: Augmentation index; AUC: Area under the curve; baPWV: Brachial-ankle pulse wave velocity; CAG: Coronary angiography; CAS: Coronary artery stenosis; CSBP: Central systolic blood pressure; DBP: Diastolic blood pressure; PCI: Percutaneous coronary artery intervention; PWV: Pulse wave velocity; ROC: Receiver operating characteristic

\section{Acknowledgements}

The authors thank Joon Won Kang and Yon Won Lee at the cardiac catheterization laboratory for their skilled technical assistance.

\section{Availability of data and materials}

Raw data supporting the obtained results are available at the corresponding author.

\section{Authors' contributions}

$\mathrm{HJ}$ and $\mathrm{DL}$ designed the study. $\mathrm{HJ}$ and $\mathrm{SJ}$ analyzed the data and drafted the first version of the manuscript. JC, JHP, SH and CY collected the data and critiqued the manuscript. All authors read and approved the final manuscript.

\section{Competing interests}

The authors declare that they have no competing interests.

\section{Consent for publication}

Not applicable.

\section{Ethics approval and consent to participate}

This study was approved by the ethical committee of the institutional review board of Korea University Anam Hospital, and the need for written informed consent was waived due to the non-interventional and retrospective nature of the study.

Received: 3 June 2016 Accepted: 16 January 2017

Published online: 31 January 2017

\section{References}

1. Oliver JJ, Webb DJ. Noninvasive assessment of arterial stiffness and risk of atherosclerotic events. Arterioscler Thromb Vasc Biol. 2003;23(4):554-66.

2. Mattace-Raso FU, van der Cammen TJ, Hofman A, van Popele NM, Bos ML, Schalekamp MA, Asmar R, Reneman RS, Hoeks AP, Breteler MM, et al. Arterial stiffness and risk of coronary heart disease and stroke: the Rotterdam Study. Circulation. 2006;113(5):657-63.

3. Boutouyrie P, Tropeano Al, Asmar R, Gautier I, Benetos A, Lacolley P, Laurent $S$. Aortic stiffness is an independent predictor of primary coronary events in hypertensive patients: a longitudinal study. Hypertension. 2002;39(1):10-5. 
4. Liao D, Arnett DK, Tyroler HA, Riley WA, Chambless LE, Szklo M, Heiss G. Arterial stiffness and the development of hypertension. The ARIC study. Hypertension. 1999;34(2):201-6.

5. Lehmann ED, Gosling RG, Sonksen PH. Arterial wall compliance in diabetes. Diabet Med. 1992;9(2):114-9.

6. Mitchell GF, Parise H, Benjamin EJ, Larson MG, Keyes MJ, Vita JA, Vasan RS, Levy D. Changes in arterial stiffness and wave reflection with advancing age in healthy men and women: the Framingham Heart Study. Hypertension. 2004;43(6):1239-45.

7. Laurent S, Cockcroft J, Van Bortel L, Boutouyrie P, Giannattasio C, Hayoz D, Pannier B, Vlachopoulos C, Wilkinson I, Struijker-Boudier H, et al. Expert consensus document on arterial stiffness: methodological issues and clinical applications. Eur Heart J. 2006;27(21):2588-605.

8. Yamashina A, Tomiyama H, Takeda K, Tsuda H, Arai T, Hirose K, Koji Y, Hori S, Yamamoto Y. Validity, reproducibility, and clinical significance of noninvasive brachial-ankle pulse wave velocity measurement. Hypertens Res. 2002;25(3):359-64.

9. Chae MJ, Jung $\mathbb{H}_{\text {, Jang }} \mathrm{DH}$, Lee $S Y$, Hyun JY, Jung JH, Ahn DS, Lim DS, Lee SJ. The brachial ankle pulse wave velocity is associated with the presence of significant coronary artery disease but not the extent. Korean Circ J. 2013; 43(4):239-45.

10. Imanishi R, Seto S, Toda G, Yoshida M, Ohtsuru A, Koide Y, Baba T, Yano K. High brachial-ankle pulse wave velocity is an independent predictor of the presence of coronary artery disease in men. Hypertens Res. 2004;27(2):71-8.

11. Izuhara M, Shioji K, Kadota S, Baba O, Takeuchi Y, Uegaito T, Mutsuo S, Matsuda M. Relationship of cardio-ankle vascular index (CAVI) to carotid and coronary arteriosclerosis. Circ J. 2008;72(11):1762-7.

12. Hope SA, Antonis P, Adam D, Cameron JD, Meredith IT. Arterial pulse wave velocity but not augmentation index is associated with coronary artery disease extent and severity: implications for arterial transfer function applicability. J Hypertens. 2007;25(10):2105-9.

13. Song BG, Park JB, Cho SJ, Lee SY, Kim JH, Choi SM, Park JH, Park YH, Choi JO, Lee SC, et al. Pulse wave velocity is more closely associated with cardiovascular risk than augmentation index in the relatively low-risk population. Heart Vessels. 2009;24(6):413-8.

14. Gensini GG. A more meaningful scoring system for determining the severity of coronary heart disease. Am J Cardiol. 1983;51(3):606.

15. Kips JG, Schutte AE, Vermeersch SJ, Huisman HW, Van Rooyen JM, Glyn MC Fourie CM, Malan L, Schutte R, Van Bortel LM, et al. Comparison of central pressure estimates obtained from SphygmoCor, Omron HEM-9000Al and carotid applanation tonometry. J Hypertens. 2011;29(6):1115-20.

16. Khan TH, Farooqui FA, Niazi K. Critical review of the ankle brachial index Curr Cardiol Rev. 2008;4(2):101-6.

17. Criqui MH, McClelland RL, McDermott MM, Allison MA, Blumenthal RS, Aboyans V, Ix JH, Burke GL, Liu K, Shea S. The ankle-brachial index and incident cardiovascular events in the MESA (Multi-Ethnic Study of Atherosclerosis). J Am Coll Cardiol. 2010;56(18):1506-12

18. Luft FC. Molecular mechanisms of arterial stiffness: new insights. J Am Soc Hypertension. 2012;6(6):436-8.

19. Weber T, Auer J, O'Rourke MF, Kvas E, Lassnig E, Berent R, Eber B. Arterial stiffness, wave reflections, and the risk of coronary artery disease. Circulation. 2004;109(2):184-9.

20. Park SM, Seo HS, Lim HE, Shin SH, Park CG, Oh DJ, Ro YM. Assessment of arterial stiffness index as a clinical parameter for atherosclerotic coronary artery disease. Circulation J. 2005;69(10):1218-22.

21. Mitchell GF, Hwang SJ, Vasan RS, Larson MG, Pencina MJ, Hamburg NM, Vita JA, Levy D, Benjamin EJ. Arterial stiffness and cardiovascular events: the Framingham Heart Study. Circulation. 2010;121(4):505-11.

22. Aatola H, Hutri-Kahonen N, Juonala M, Viikari JS, Hulkkonen J, Laitinen T, Taittonen L, Lehtimaki T, Raitakari OT, Kahonen M. Lifetime risk factors and arterial pulse wave velocity in adulthood: the cardiovascular risk in young Finns study. Hypertension. 2010;55(3):806-11.

23. Mancia G, Fagard R, Narkiewicz K, Redon J, Zanchetti A, Bohm M, Christiaens T, Cifkova R, De Backer G, Dominiczak A, et al. 2013 ESH/ESC quidelines for the management of arterial hypertension: the Task Force for the Management of Arterial Hypertension of the European Society of Hypertension (ESH) and of the European Society of Cardiology (ESC). Eur Heart J. 2013;34(28):2159-219.

24. Xiong Z, Zhu C, Zheng Z, Wang M, Wu Z, Chen L, Chen Y. Relationship between arterial stiffness assessed by brachial-ankle pulse wave velocity and coronary artery disease severity assessed by the SYNTAX score. J Atheroscler Thromb. 2012;19(11):970-6.
25. Seo WW, Chang HJ, Cho I, Yoon YY, Suh JW, Kim Kl, Cho YS, Youn TJ, Chae $\mathrm{IH}$, Choi DJ, et al. The value of brachial-ankle pulse wave velocity as a predictor of coronary artery disease in high-risk patients. Korean Circulation J. 2010;40(5):224-9.

26. Park KH, Kim MK, Kim HS, Park WJ, Cho GY, Choi YJ. Clinical significance of framingham risk score, flow-mediated dilation and pulse wave velocity in patients with stable angina. Circulation J. 2011;75(5):1177-83.

27. Park H, Das M, Aronow WS, McClung JA, Belkin RN. Relation of decreased ankle-brachial index to prevalence of atherosclerotic risk factors, coronary artery disease, aortic valve calcium, and mitral annular calcium. Am J Cardiol. 2005;95(8):1005-6.

28. Zheng ZJ, Sharrett AR, Chambless LE, Rosamond WD, Nieto FJ, Sheps DS, Dobs A, Evans GW, Heiss G. Associations of ankle-brachial index with clinical coronary heart disease, stroke and preclinical carotid and popliteal atherosclerosis: the Atherosclerosis Risk in Communities (ARIC) Study. Atherosclerosis. 1997;131(1): $115-25$.

29. Sadeghi M, Heidari R, Mostanfar B, Tavassoli A, Roghani F, Yazdekhasti S. The Relation Between Ankle-Brachial Index (ABI) and coronary artery disease severity and risk factors: an angiographic study. ARYA Atheroscler. 2011;7(2):68-73.

30. Falcao FJ, Rodrigues Alves CM, Caixeta A, de Freitas Guimaraes L, de Sousa Filho JT, Soares JA, Helber I, Carvalho AC. Relation between the anklebrachial index and the complexity of coronary artery disease in older patients. Clin Interv Aging. 2013;8:1611-6.

31. Papamichael CM, Lekakis JP, Stamatelopoulos KS, Papaioannou TG, Alevizaki MK, Cimponeriu AT, Kanakakis JE, Papapanagiotou A, Kalofoutis AT, Stamatelopoulos SF. Ankle-brachial index as a predictor of the extent of coronary atherosclerosis and cardiovascular events in patients with coronary artery disease. Am J Cardiol. 2000;86(6):615-8.

\section{Submit your next manuscript to BioMed Central and we will help you at every step:}

- We accept pre-submission inquiries

- Our selector tool helps you to find the most relevant journal

- We provide round the clock customer support

- Convenient online submission

- Thorough peer review

- Inclusion in PubMed and all major indexing services

- Maximum visibility for your research

Submit your manuscript at www.biomedcentral.com/submit
) Biomed Central 